\title{
HIDROLOGIA DEL ALTO GOLFO DE CALIFORNIA II. CONDICIONES DURANTE INVIERNO. PRIMAVERA Y VERANO.
}

\author{
por: \\ Saúl Alvarez Borrego* \\ Bernardo Primitivo Flores Báez y Luis Arnulfo Galindo Bect
}

* Ocupación Actual: Investigador del Centro de Investigación Científica y Educación Superior de Ensenada, B. C.

\section{RESUMEN}

Durante invierno, primavera y verano de 1973 se realizaron ocho cruceros hidrográficos en el Alto Golfo de California. Se realizaron determinacianes de temperatu$\mathrm{ra}$, salinidad, concentración de oxígeno disuelto, $\mathrm{pH}$ y condiciones meteorológicas. Esto complementa los resultados presentados por Alvarez Borrego y Galindo Bect (1974) para otoño de 1972, cerrando el ciclo anual de variación de las condiciones hidrológicas. Existe una gran variabilidad en el Alto Golfo de California, con temperatura minima de $8.25^{\circ} \mathrm{C}$, registrada en diciembre, y máxima de $32.58^{\circ} \mathrm{C}$, registrada en agosto; con salinidad superficial mínima de $35.28 \%$, registrada en ociubre, y máxima de $41.00 \%$, en julio. No existe ningún aporte significativo de agua dulce del Río Colorado en todo el año. Esto está demostrado por las elevadas salinidades registradas en lo que fuera la boca del Rín; con excepción de los días en que se registra una precipitación pluvial local relativamente considerable, lo cual sucede muy pocos días del año. El gradiente superficial de temperatura sufre reversiones al principio de primavera y de otoño, debido al ciclo anual de la irradiación solar y la temperafura atmosférica. Sin embargo, la salinidad mantiene en general el mismo gradiente con los valore's aumentando hacia el noroeste.

\section{ABSTRACT}

Eight cruises were carried on during winter, spring and summer of 1973, in the northern end of the Gulf af California. Determinations of temperature, salinity, dissolved oxygen concentration, $\mathrm{pH}$ and meteorological conditions were made. This is the continuation of the study presented by Alvarez Borrego and Galindo Bect (1974) on the fall conditions; completing the annual cycle. A great variability exists at the head of the Gulf, with minimum temperature of $8.25^{\circ} \mathrm{C}$ registered in December, and maximum of $32.58^{\circ} \mathrm{C}$ in August; with minimum surface salinity of $35.28 \%$, registred in October, and maximum of $41.00 \%$ in July. There is no significant input of fresh water from the Colorado river to the Gulf during all year. This is demonstrated by the high salinities registered at what used to be the river mouth; with exception of the very few days when local rain is relatively high. The surface temperature gradient reverses at the beginning of spring and fall, due to the annual cycle of solar radiation and atmospheric temperature. Salinity maintains in general the same gradient, with values increasing northwestwards.

\section{INTRODUCCION}

El Alto Golfo de California tiene una forma más o menos triangular y se encuentra localizado al norte de los $31^{\circ} \mathrm{N}$. Al oeste se encuentra Baja California y al este Sonora. La costa oeste está for- mada de grandes marismas lodosas, cuyos sedimentos se derivan del Río Colorado (Thompson, 1969). Al lado noreste se encuentra el desierto de Sonora o desierto de El Altar. Cerca de la cossta, el 
desierta consiste de un plano aluvial de arena y grava que en ciertos lugares está cubierto de dunas de arena (Schreiber, Jr., 196\%1. Esta área se ha clasificado como una de las porciones más áridas del desierto norteamericano (Meigs, 1953)

Las islas de Tiburón y Angel de la Guarda, localizadas en la parte media del Golfo, actúan como disipadores para la mayoría de los ciclones que entran al Golfo de California (Roden, 1964). La velocidad máxima de viento. registrada en las instalaciones de la Universidad de Sonora y la Universidad de Arizona en Puerto Peñasco, Son., ha sido de 76 $\mathrm{mi} / \mathrm{h}$. durante el otoño de 1967 (Green. 1969).

Las mareas son de gran amplitud en el Alto Golfo de California, con compow nentes diurnos y semidiurnos. Los componentes diurnos aumentan ligeramente de la boca del Golfo hacia el norte. y los componentes semidiurnos disminuyen (Mathews, 1969). Recientemente se ha contado con calendarios para predicción de mareas en el norte del Golfo (Mathews, Thomson y Browning, 1967).

El fondo del Alto Golfo de California tiene una topografía muy irregular, con una serie de canales y bajos con dirección noroeste-sureste, mejor desarrollados hacia lo que fue la boca del Río Colorado. Esto dificulta la navegación de embarcaciones con calado mayor de un metro, sobre todo cuando no se tiene familiaridad con las fluctuaciones de mareas y las fuertes corrientes provocadas por las mismas.

Desde 1935 el flujo del río ha sido atrapado en el lago Mead y a medida que se descarga en la sección del río aguas abajo de este lago, se retiene en la parte superior del delta donde se utiliza para fines agrícolas. La causa principal del cambio del flujo ha sido la construcción de presas, particularmente la presa Hoover (Schreiber Jr., 1969). Los resultados del presente trabajo indican que solamente en los días en que se registra precipitación pluvial existe aporte de agua duce al Alto Golfo de California, el cual es de origen local.

Alvarez Borrego y Galindo Bect (1974) discutieron las condiciones hidrológicas del Alto Golfo para otoño. El presente trabajo es una continuación del anterior, de tal manera que se completa un ciclo anual.

\section{OBTENCION DE DATOS}

Se realizaron ocho cruceros oceanográficos en el período de Enero a Octubre de 1973. Las fechas de estos cruceros fueron: 16-19 de Enero, 1-2 de Febrero, 9-14 de Marzo, 21-24 de Mayo, 26-27 de Junio, 21-22 de Julio, 17-18 de Agosto y del 30 de Septiembre al 2 de Octubre. Para los cruceros de Julio y Agosto se utilizaron barcos camaroneros de Puerto Peñasco, Son. Para el resto de los cruceros se utilizó el "Adventyr" de 44 pies de eslora.

La localización de las estaciones hidrográficas se muestran en la figura 1. Las estaciones representadas por círculos oscuros, de la figura $1^{a}$ se ocuparon en los cruceros de Febrero, Mayo, Junio, Julio, Agosto y Septiembre-Oçtubre; en Marzo se ocuparon además las estaciones representadas por círculos claros. El patrón de estaciones de la figura lb se cubrió en el crucero de Enero. En este crucero se cambió la posición de las estaciones para trabajar en conjunto con el personal de la Universidad de Arizona.

Los métodos de muestreo y análisis fueron los mismos de Alvarez Borrego y Galindo Bect (1974). En los cruceros de Enero, Marzo y Agosto, no se determinó el $\mathrm{pH}$ debido a fallas en el potenciómetro. Con el fin de conocer la variación diurna de los diferentes parámetros, se realizaron determinaciones de los mismos durante 24 horas, cada 2 horas, en dos ocasiones: Marzo y Mayo. El estudio de la variacion diurna de Marzo se realizó en la estación A-6 (Fig. la) y el de Mayo frente a Santa Clara, Son.

\section{RESULTADOS}

\section{a) Temperatura.}

Eñ Enero y Febrero, la temperatura superficial disminuyó en general de este a oeste (Fig. 2a y b). Las temperaturas mínimas en estos dos meses se registraron al oeste de isla Montague, y fueron $12.21^{\circ} \mathrm{C}$ en Enero y $10.85^{\circ} \mathrm{C}$ en Fe- 

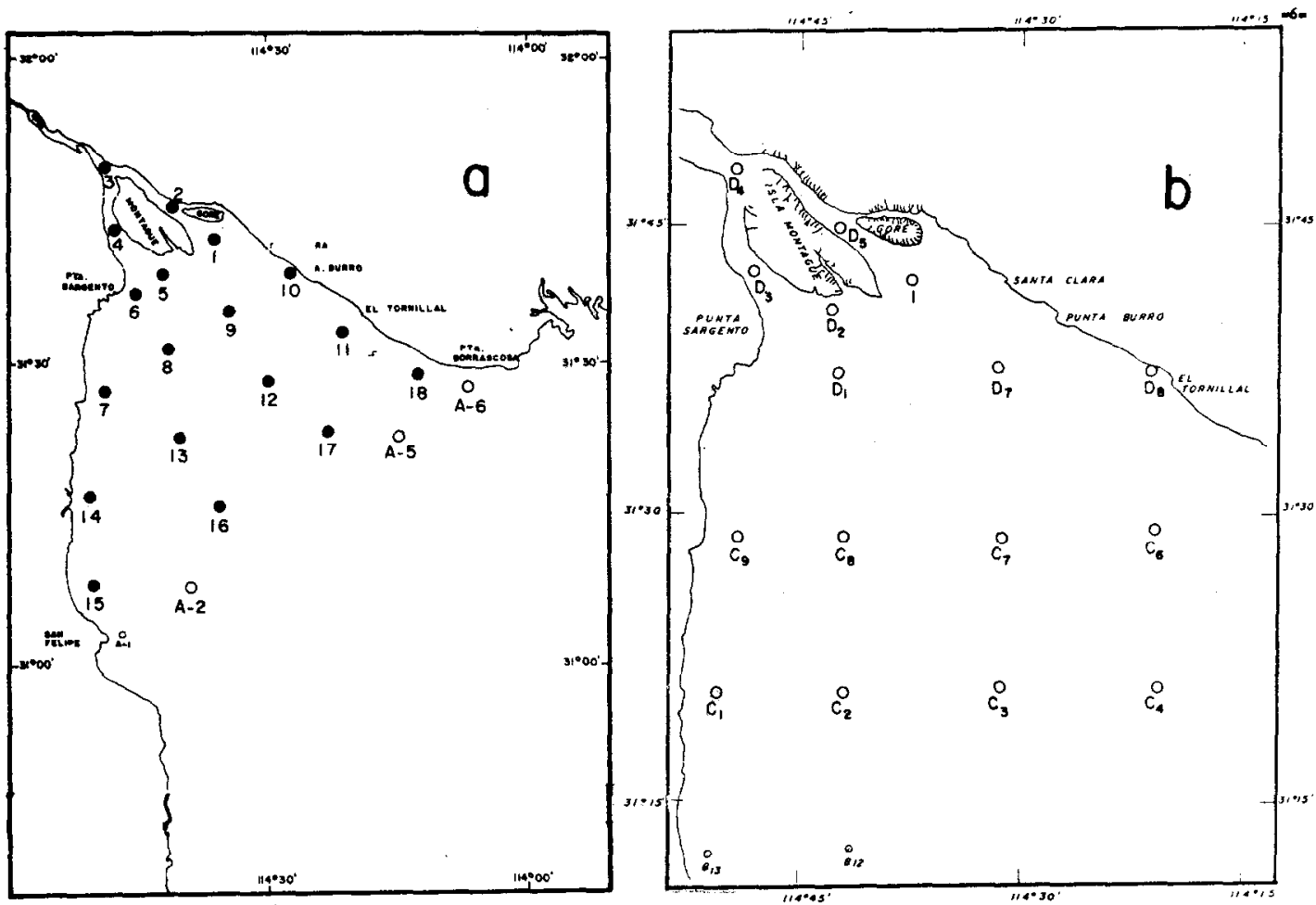

Fig. I. Localización de las estaciones hidrográficas. a. Para Febrero, Marzo. Mayo, Junio, Julio, Agosto y SepriembreOctubre. b. Para Enero.

brero. En Marzo la distribución de temperatura fue muy irregular, sin presentar un gradiente monotónico (Fig. 2c). La temperatura mínima se registró al norte de isla Montague en este mes y fue $16.40^{\circ} \mathrm{C}$. En Mayo, Junio, Julio y Agosto, la temperatura disminuyó en general del noroeste al sureste, contrario a la situación de Enero y Febrero (Fig. 2d,3a, b y c). Mientras que en el crucero de Septiembre-Octubre se observó de nuevo la inversión con los valores disminuyendo en general hacia el noroeste (Fig. 3d). Los valores máximos registrados en Mayo, Junio, Julio y Agosto, fueron, respectivamente, $28.80^{\circ} \mathrm{C}, 31.50^{\circ} \mathrm{C} 31.30^{\circ} \mathrm{C}$ y $32.58^{\circ} \mathrm{C}$. Esta última se registró al norte de isla Montague. La distribución superficial de temperatura fue muy regular en Mayo, pero en Junio, Julio, Agosto y Septiembre-Octubre fueron irregulares, con las isotermas en forma concéntrica o de lenguetas.

\section{b) Salinidad.}

Contrario a la distribución superficial de temperatura, la de salinidad no muestra una inversión en función de la estación del ah̆o, sino generalmente presenta la misma variación monotónica con los valores aumentando de sureste a noroeste \{Figs, 4a, b, $c, d$ y $5 a, b, c, d$ ). Los valores máximos fueron: $37.44 \%$ en Enero, $37.58^{\circ} / 00$ en Febrero, $39.99 \%$ en Marzo, $39.76 \%$ en Mayo, $39.31 \%$ oo en Junio, $41,00 \%$ en Julio, 39.66\%,00 en Agosto y $\mathbf{3 9 . 7 9 \%} / 00$ en SeptiembreOctubre. En la mayoría de los casos, estos máximos se registraron al norte de isla Montague. En Enero y Febrero las isohalinas presentaron una dirección meridional con los valores aumentando de este a oeste (Fig. 4a y b). En Marzo la distribución de salinidad fue un poco irregular; las isohalinas formaron meandros en la parte central, quizá debido a la precipitación pluvial registrada duran- 
ALVAREZ - FLORES - GALINDO
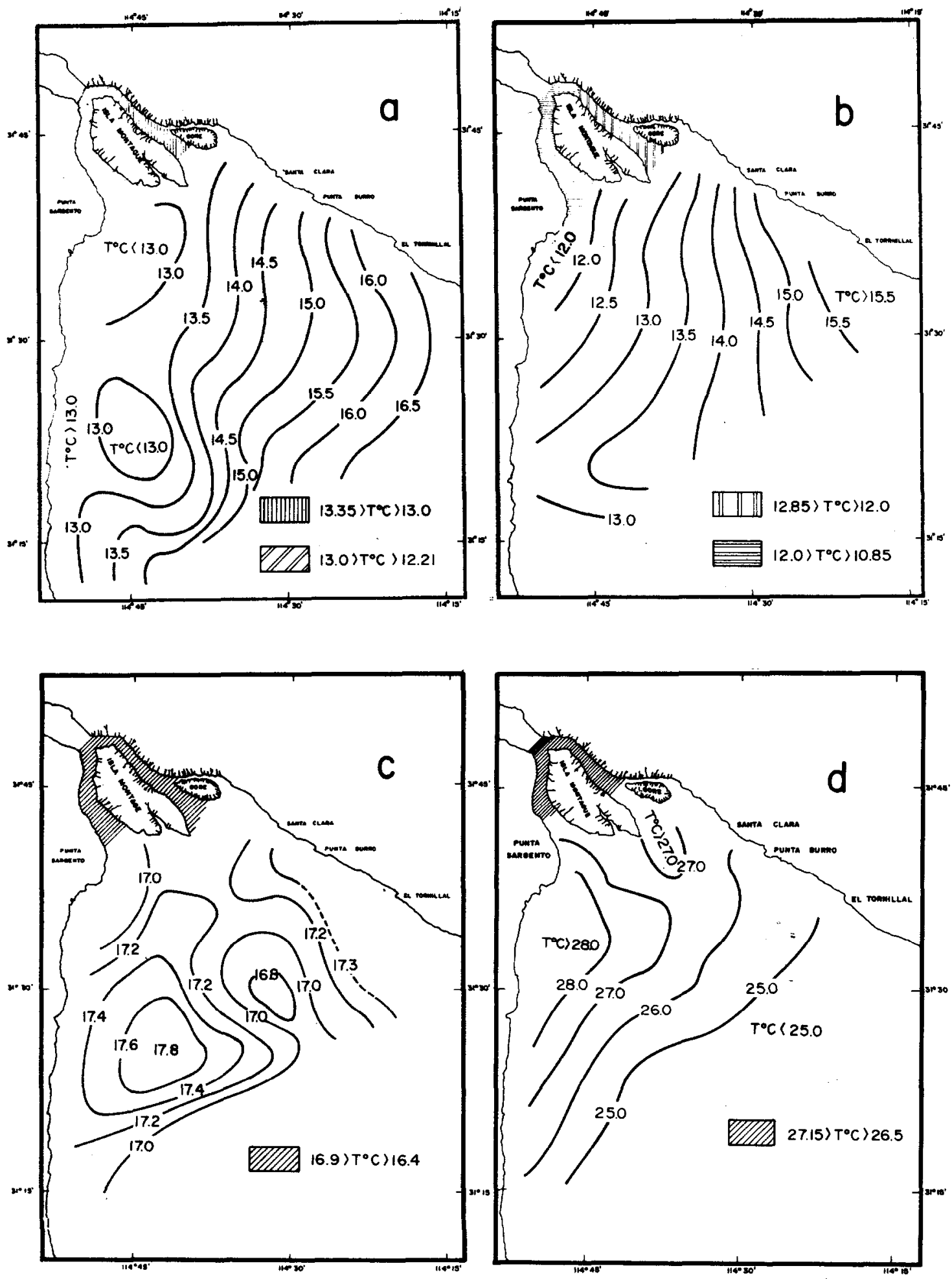

Fig. 2. Distribuciones Superficiales de Temperatura $1^{\circ} \mathrm{O}$ para a.. Enero, b. Febrero: co Mazo, d. Mere. 
te el crucero. De Mayo a Septiembre-Octubre, la distribución fue muy uniforme (Figs. 4d y $5 a, b, c$ y d).

\section{c) Concentración de oxígeno disuélto.}

La' distribución superficial de oxígeno no es tan uniforme, en los diferentes meses del año, como la de temperatura y la de salinidad. En Enero, la concentración de oxígeno presentó en general una tendencia de aumentar de sureste a noroeste (Fig. 6a); esto es en concordancia con la distribución de temperatura en este mes, ya que a menor temperatura mayor solubilidad de los gases. El valor máximo registrado en este crucero fue $6.92 \mathrm{ml} / \mathrm{l}$, al este de isla Montague. En Febrero la distribución de oxígeno presentó una disminución de valores de la parte central del área de estudio hacia el noroeste y hacia el sureste; y un aumento hacia el noreste y hacia el suroeste (Fig. 6b). El valor mínimo registrado en Febrero fue $5.60 \mathrm{ml} / \mathrm{l}$, al norte de isla Montague; y el máximo fue 7.20 $\mathrm{ml} / \mathrm{l}$, cerca de Punta Burro, Son. En Marzo, la distribución de oxígeno mostró un centro de valores altos desde el cual los valores disminuyeron hacia todas direcciones en el área de estudio (Fig. 6c). El mínimo fue $5.20 \mathrm{ml} / \mathrm{l}$ y se registró cerca de la costa de Sonora, al sur de isla Gore; y el máximo fue $6.70 \mathrm{ml} / \mathrm{l}$, en el centro mencionado. En Mayo, los valores de concentración de oxígeno disuelto disminuyeron de sureste a noroeste, en concordancia con la distribución de temperatura (Fig. 2d y $6 d$ ). El mánimo fue $3.88 \mathrm{ml} / 1$ y se registró al norte de isla Montague. En Junio se presentó una distribución de oxígeno diferente a la de otros meses del año, en el sentido de que el gradiente se encontraba orientado de suroeste a noreste, con los valores aumentando al noreste (Fig. 7a). En los cruceros de Julio, Agosto y Septiembre-Octubre, la distribución de oxígeno se presentó muy compleja, sin gradientes bien establecidos (Fig. 7b, $c$ y d). En Julio se presentó un centro de valores altos en la parte suroriental de la zona de estudio, y un centro de valores bajos más al norte (Fig. $7 \mathrm{~b}$ ). En Agosto se presentó una lengueta de valores altos, originándose en lo parte cercana a Baia Colifornia ira. 7c: En Sertembroctubls ia dis- tribución de oxígeno fue menos irregular que en los meses de Verano; con un centro de altos valores en la parte central (Fig. 7d). El mínimo registrado en el crucero de Septiembre-Octubre, que fue el más bajo de Verano y principios de Otoño, fue $3.33 \mathrm{ml} / \mathrm{l}$, al norte de isla Montague.

d) $\mathbf{p H}$.

En los cruceros de Enero, Marzo y Agosto no se obtuvieron datos de $\mathrm{pH}$ debido a desperfectos en el potenciómetro. En general la distribución superficial de $\mathrm{pH}$ para los diferentes meses es bastante consistente con la distribución de oxígeno (Figuras $6 b$ y $d ; 7 a, b$ y $d ; 8 a, b$, c y d; y 91; con valores altos de oxígeno correspondiendo a valores altos de pti y viceversa.

\section{e) Variación diurna.}

En el crucero de Marzo se estudió la variación de temperatura, salinidad y concentación de oxígeno disuelto en un ciclo de 24 horas, de las 11:00 A.M. del 9 de Marzo a las 11:00 A.M. del 10 de Marzo (Fig. 10a). Este estudio se realizó frente a Punta Borrascosa, Son. (Estación A-6, Fig. la). Se presentaron bien marcados un mínimo y un máximo de salinidad. El máximo fue $36.19 \%$ y se presentó a las 13:00 Hrs., y el mínimo fue $35.80 \%$ y se presentó a las 19:00 Hrs. y a las 05:00 Hrs. (Fig. 10a). Entre las 19:00 Hrs. y las 05:00 Hrs. se registró un pequeño aumento en la salinidad, alcanzando un valor de $35.83 \%$; este incremento se considera real ya que el salinómetro tiene una precisión de

$0.005 \%$. La temperatura tuvo un comportamiento un poco menos regular que el de la salinidad; presentó un máximo de $17.60^{\circ} \mathrm{C}$ arlas 17:00 Hrs., y un minimo de $17.10^{\circ} \mathrm{C}$ a las 07:00 Hrs.; entre las 03:00 Hrs. y las 05:00 Hrs. hubo un aumento de $0.10^{\circ} \mathrm{C}$ para disminuir después hasta el mínimo mencionado. La concentración de oxígeno disuelto presentó una variación más compleja, con 3 mínimos y 2 máximos. Los mínimos fueron: $5.20 \mathrm{ml} / \mathrm{l}, 5.47 \mathrm{ml} / \mathrm{l}$ y $5.42 \mathrm{ml} / 1, \mathrm{y}$ se registraran a lás 11:00 Hrs., 23:00 Hrs. y 05:00-07:00 Hrs., respectivamente. Los méximos fueron $5.64 \mathrm{ml} / \mathrm{l}$ y 5.70 $\mathrm{m} / \mathrm{l}$ y se registraron a las 19:00 Hrs. y $03.09 \mathrm{Hrs}$., respectivomente. 
ALVAREZ - FlORES - GALNDO
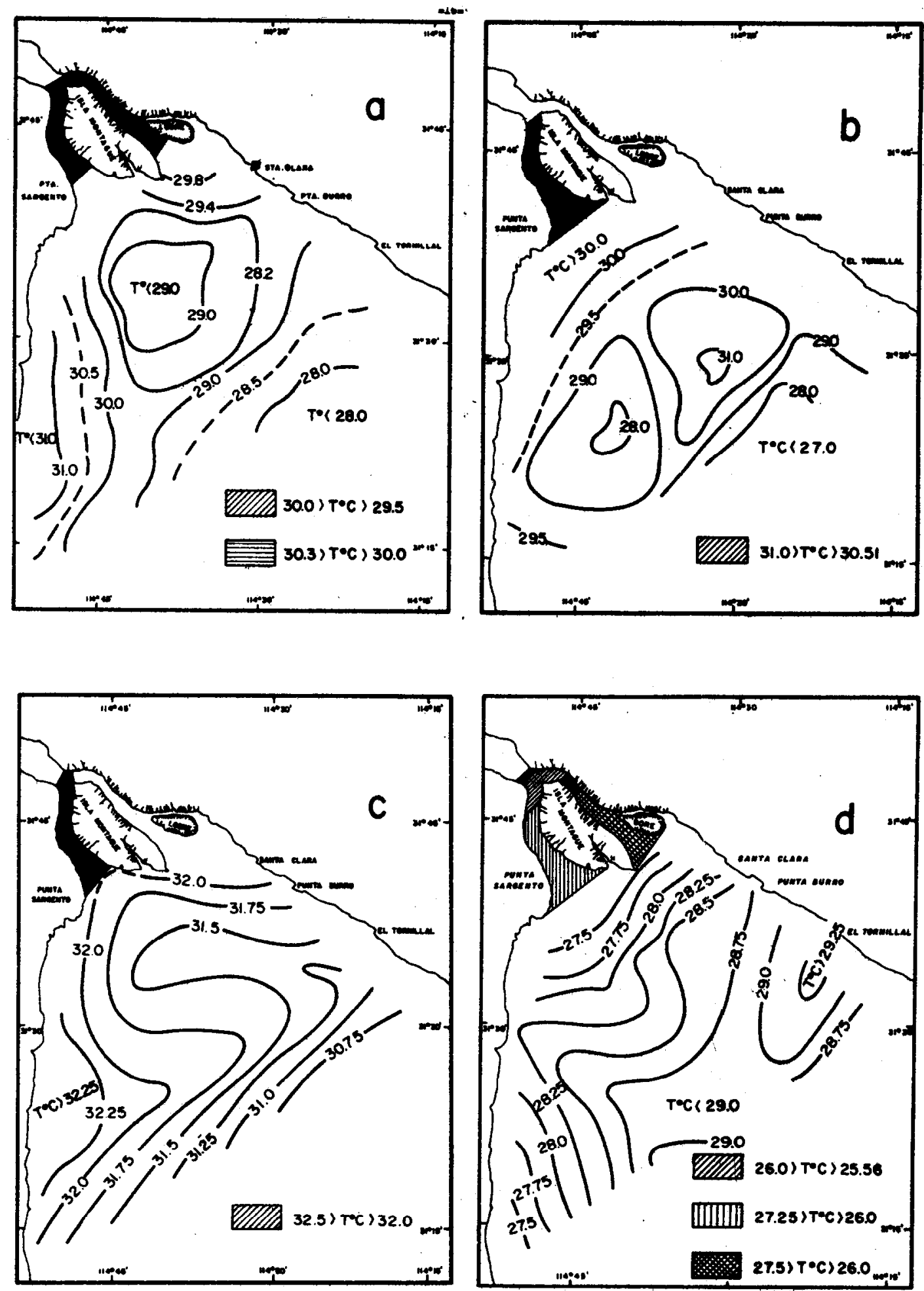

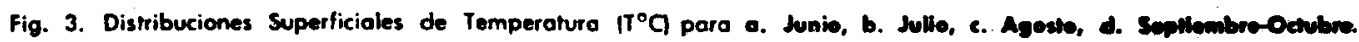



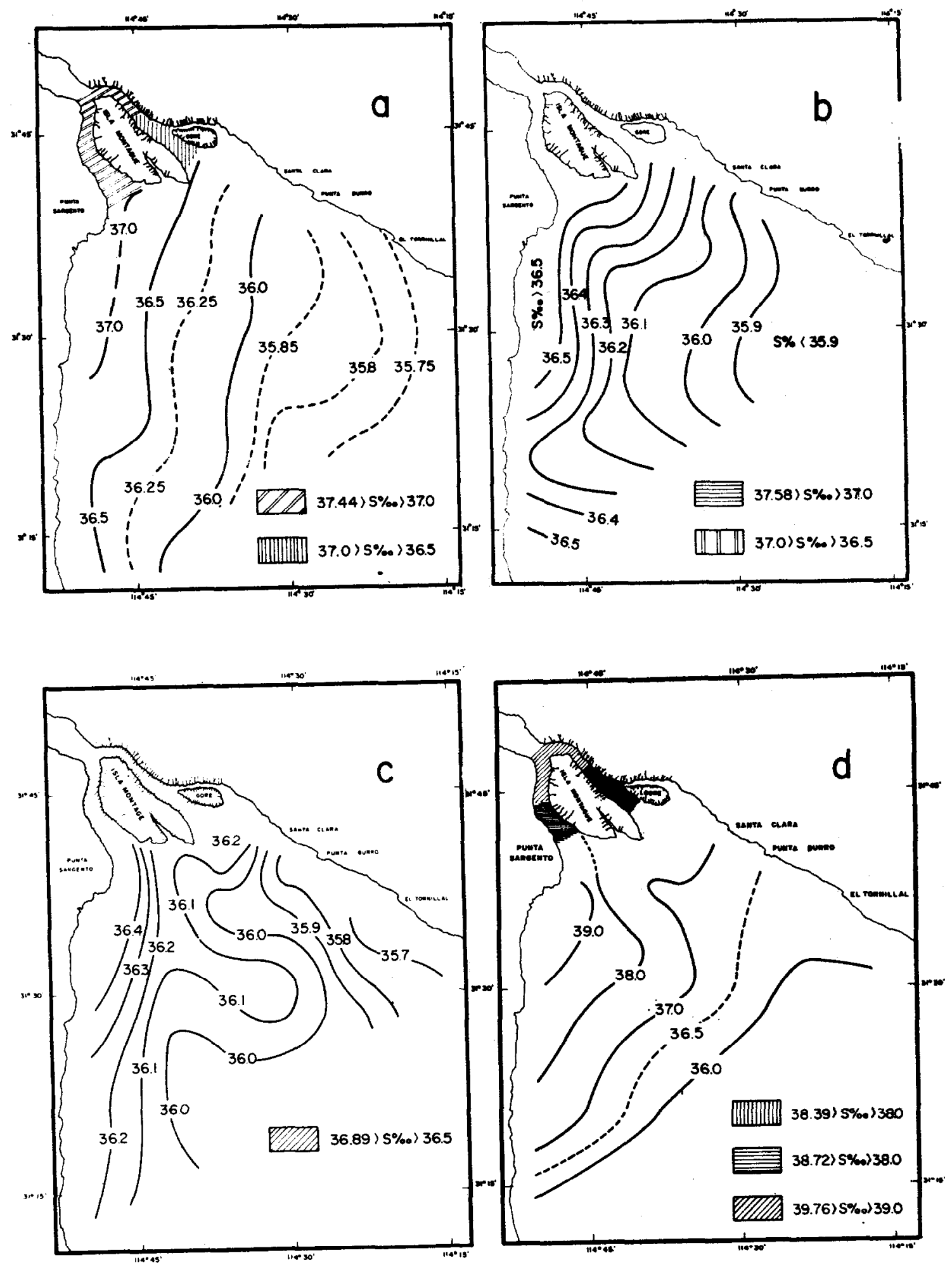

Fig. 4. Distribuciones superficiales de solinidad $\left(5^{n} / 00\right)$ para: a. Enero, b. Fabrero, c. Marzo, d. Mayo. 
En el crucero de Mayo se estudió de nuevo la variación de temperatura, salinidad y concentración de oxígeno disuelto en un ciclo de 24 horas, incluyendo además $\mathrm{pH}$; frente a Santa Clara, Son., de las 12:00 Hrs. del día 23 a las 12:00 Hrs. del 24 (Fig. 10b). El máximo de salinidad fue 36.94 y se registró a las 24:00 Hrs.; y el mínimo fue $36.74 \%$ y se registró a las 04:00 Hrs. Entre las 12:00 y las 20:00 Hrs. se registró una variación muy ligera alrededor de $36.80 \%$ oo. En general se registró un rango de salinidad menor que en el crucero de Marzo. El rango de temperatura fue mayor en Mayo.que en Marzo (Figs. 10a y b). El máximo de temperatura registrado en Mayo fue $26.80^{\circ} \mathrm{C}$ y el mínimo fue $25.70^{\circ} \mathrm{C}$, a las $14: 00 \mathrm{Hrs}$. y $10 \mathrm{Hrs}$. respectivamente. La variación diurna de la concentración de oxígeno disuelto fue menos compleja en Mayo que en Marzo. En Mayo se registraron dos máximos de oxígeno, a las 16:00 $\mathrm{Hrs}$. del día 23 y a las 12:00 del 24, con valores de 5.04 $\mathrm{ml} / \mathrm{l}$ y $5.06 \mathrm{ml} / \mathrm{l}$ respectivamente; $\mathrm{y}$ el mínimo, de $4.59 \mathrm{ml} / \mathrm{l}$, se registró a Jas 06:00 Hrs. El pH no presentó una correlación elevada con el oxígeno; se mantuvo constante de las 18:00 Hrs. a las 04:00 Hrs. El rango fue salamente de una décima de unidad de $\mathrm{pH}$.

\section{DISCUSIONES}

Alvarez Borrego y Galindo Bect (1974), al discutir los resultados obtenidos en otoño, enfatizan la variabilidad de las - condiciones hidrológicas del Alto Golfo de California. Los resultados obtenidos para invierno, primavera y verano, confirman esta gran variabilidad, sobre todo en cuanto a temperatura se refiere, dejando bien claro que esta zona está grandemente influenciada por el clima de los desiertos que la rodean. De especial interés es la inversión que sufre el gradiente de temperatura, con los valores aumentando en general hacia el noroeste en verano y disminuyendo en la misma dirección en invierno. Estas inversiones se realizaron en Marzo y Septiembre, y fueron causadas por el ciclo climático del área. Las temperaturas mínima y máxima, $8.25^{\circ} \mathrm{C}$ en Diciembre (Alvarez Eorrego yolindo 9ent, 1974 ) y $32.58^{\circ} \mathrm{C}$ en Agosto, respectivamente, se registraron en las aguas adyacentes a isla Montague, que son las más someras de la zona, y por lo tanto las más afectadas por la temperatura atmosférica.

Con excepciones muy particulares $y$ de corta duración, como la situación reportada por Alvarez Borrego y Galindo Bect (1974) para Octubre de 1972, el Alto Golfo de California es una zona antiestuarina, con el gradiente de salinidad generalmente monotónico, y los valores de la misma aumentando hacia las áreas de baja profundidad. Las más altas salinidades siempre se registraron en las aguas adyacentes a isla Montague, de invierno a verano, lo cual demuestra que no hay aporte significativo de agua dulce del Río Colorado. La evaporación en esta zona es mayor que cualquier aporte de agua dulce. Las elevadas salinidades en la parte norte, con valores hasta de más de $37.40^{\circ}$ oo en. Diciembre (Alvarez Borrego y Galindo Bect, 1974), Enero y Febrero (Figs. $4 a$ y b), indican que aún en invierno, con temperaturas atmosféricás bajas, la evaporación es considerable. Solamente en Octubre de 1972 se registraron salinidades relativamente menores en lo que fuera la boca del Río Colorado, y se debieron a las lluvias locales que hubo en los días que precedieron al muestreo (Alvarez Borrego y Galindo Bect, 1974). La mínima salinidad en Octubre fue $35.28 \%$ oo (Alvárez Borrego y Galindo Bect, 1974), y la máxima, registrada en Julio, también en lo que fuera la boca del Río Colorado, fue $41.00 \%$.00.

Con relación a los planes que existían para construir una planta nuclear de doble propósito, de desalinizacién y productora de energía eléctrica, en algún lugar cercano a Santa Clara, Son. (IAEA, 1968), y que produciría un afluente de desecho de alta temperatura y salinidad que posiblemente se canalizaría de retorno al mar; es necesario considerar seriamente el efecto que produciría, sobre todo en verano, en la zona de veda permanente, con salinidades y temperaturas de por sí ya muy elevadas desde que se obstruyó por completo la afluencia de agua dulce del Río Colorado. La zona adyacente a lo que fuera la boca del Río Colorado, es área de reproducción 
HIDROLOGIA DEL ALTO GOLFO DE CALIFORNIA
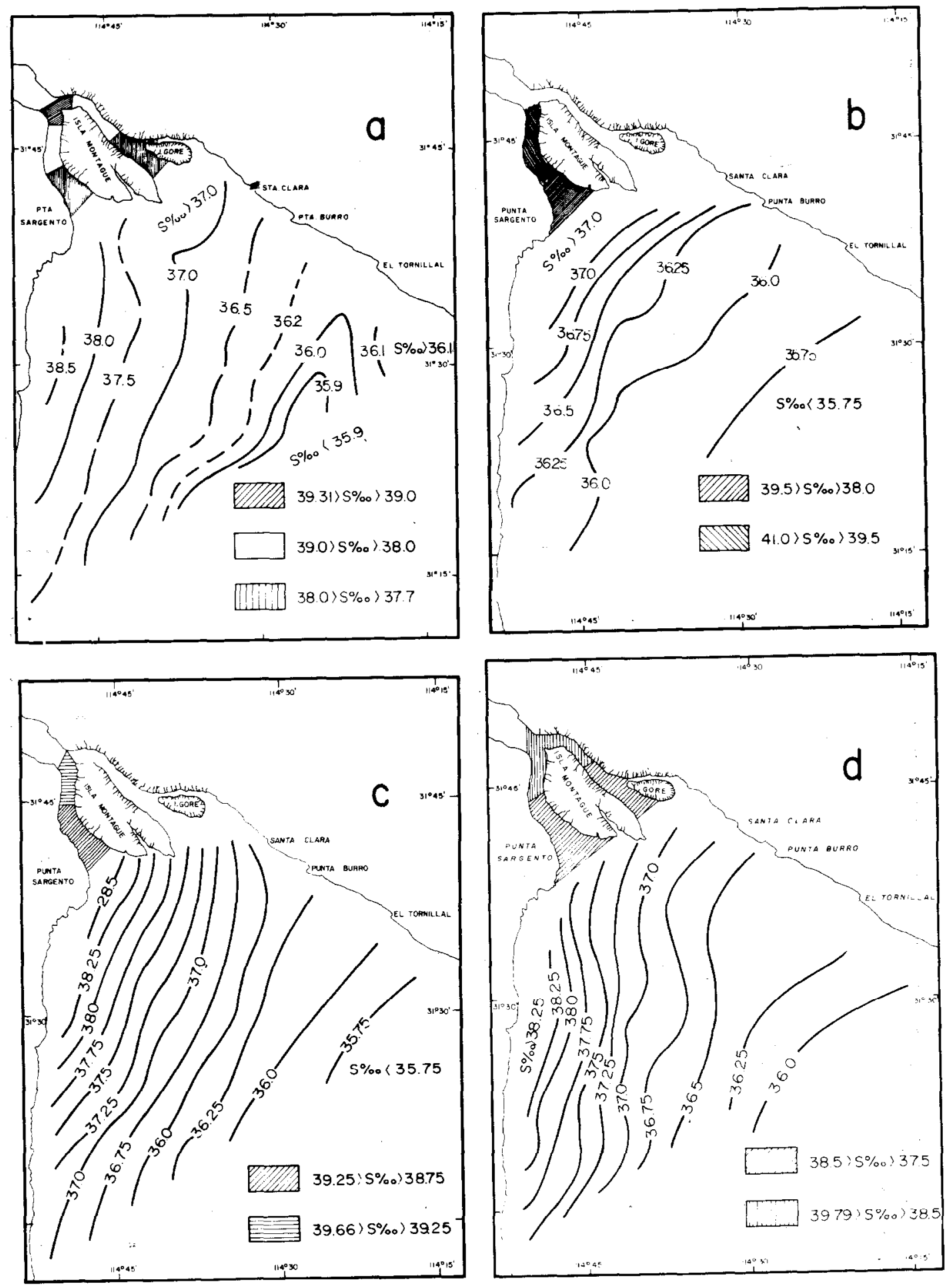

Fig. 5. Distribuciones superficiales de Solinidad $\left(S^{\prime \prime} / 00\right)$ para: a. Junio, b. Julio, c. Agosto, d. Septiembre-Octubre. 

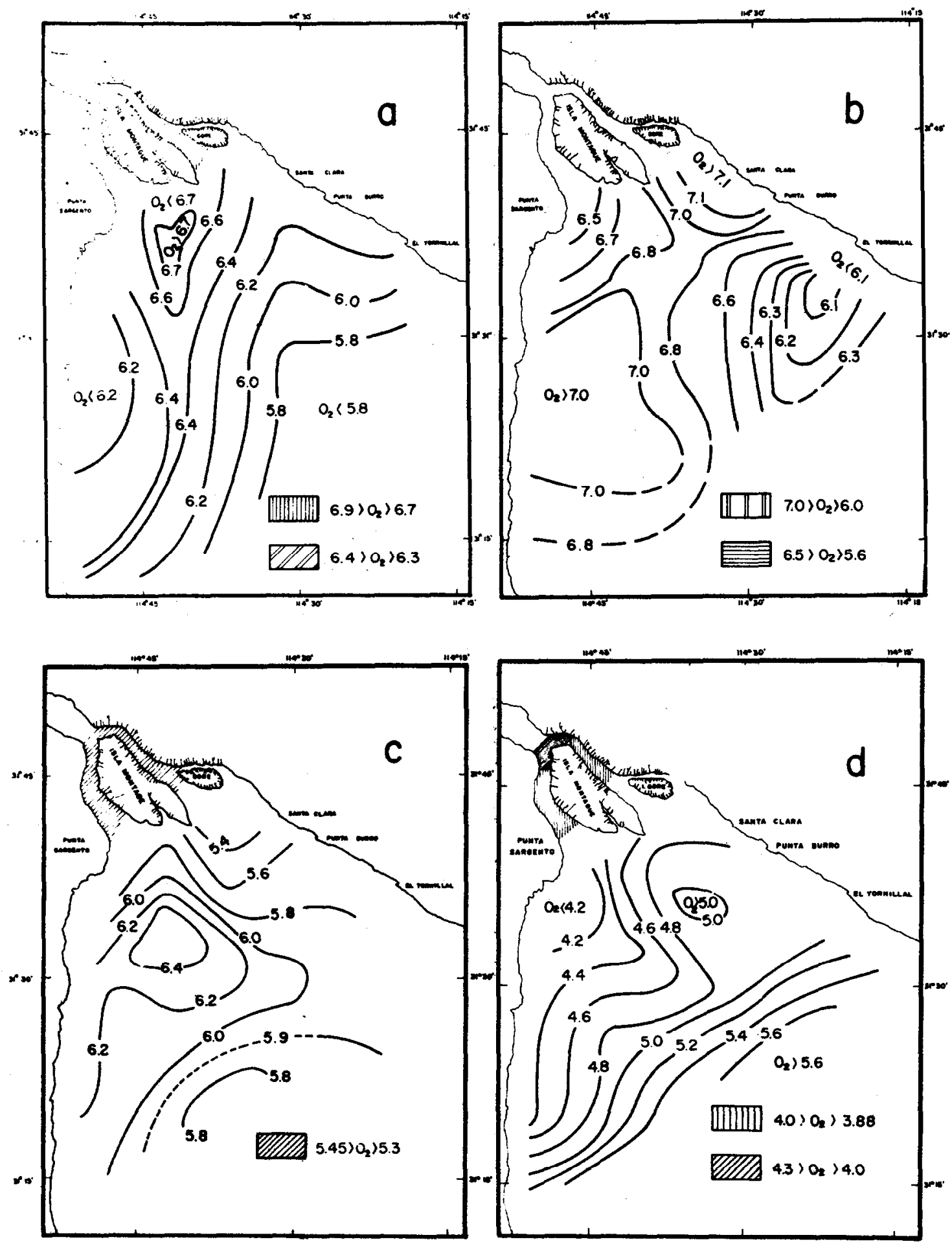

Fig. 6. Distribuciones superficiales de la concentración de oxigeno disuelto (O2) poto: o. Enaro; b. Febrero, C. Marzo, d. Movo. 
HIDROLOGIA DEL ALTO GOLFO DE CALFORNIA
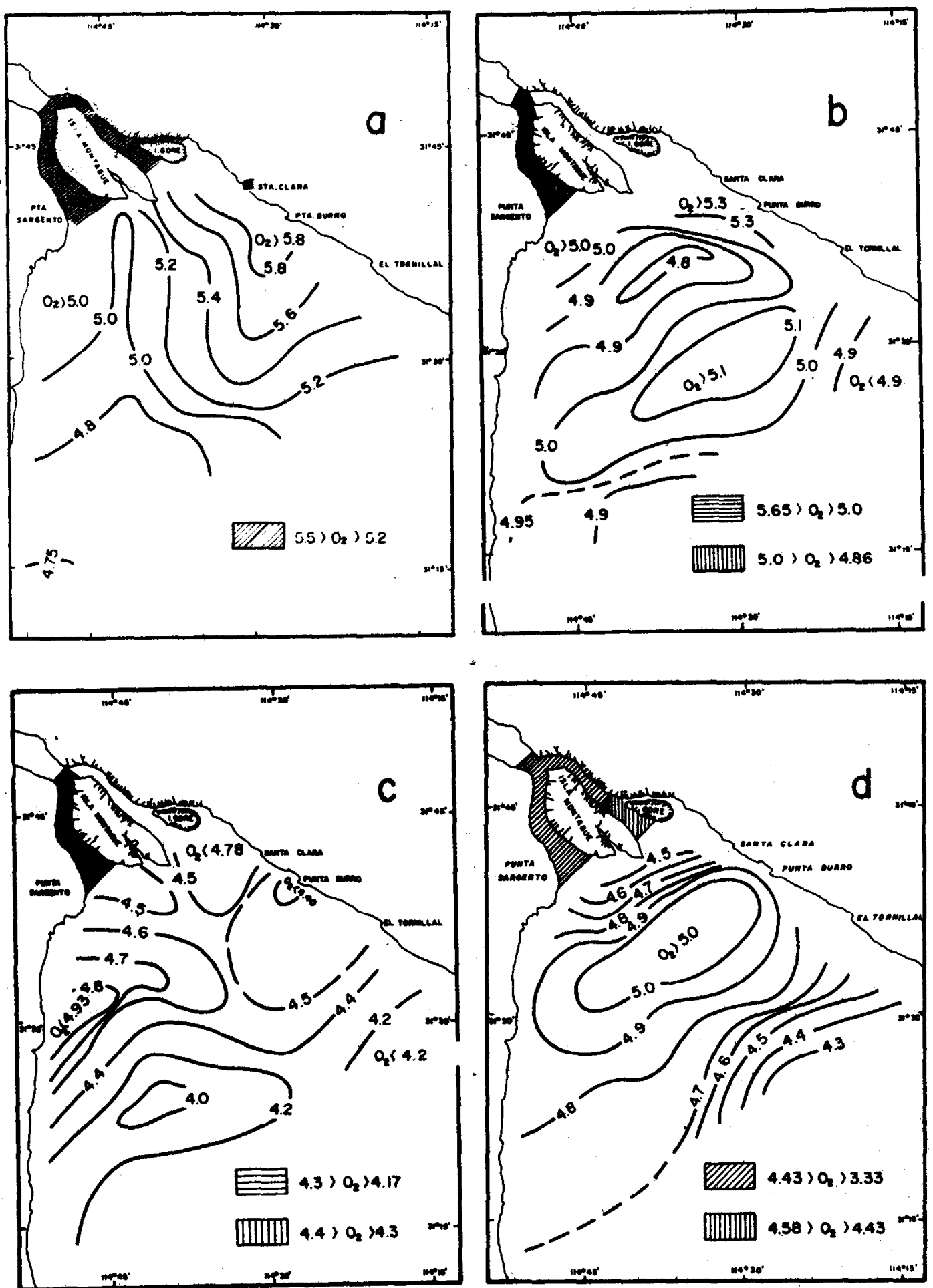

Fig. 7. Distribuciones Superficiales de lo Concentroción de Oxigeno disuelto $10^{*}$ ) poro: o. Junio, B. Julie, e. Agesto. d. Soptiembro-Oetubre. 

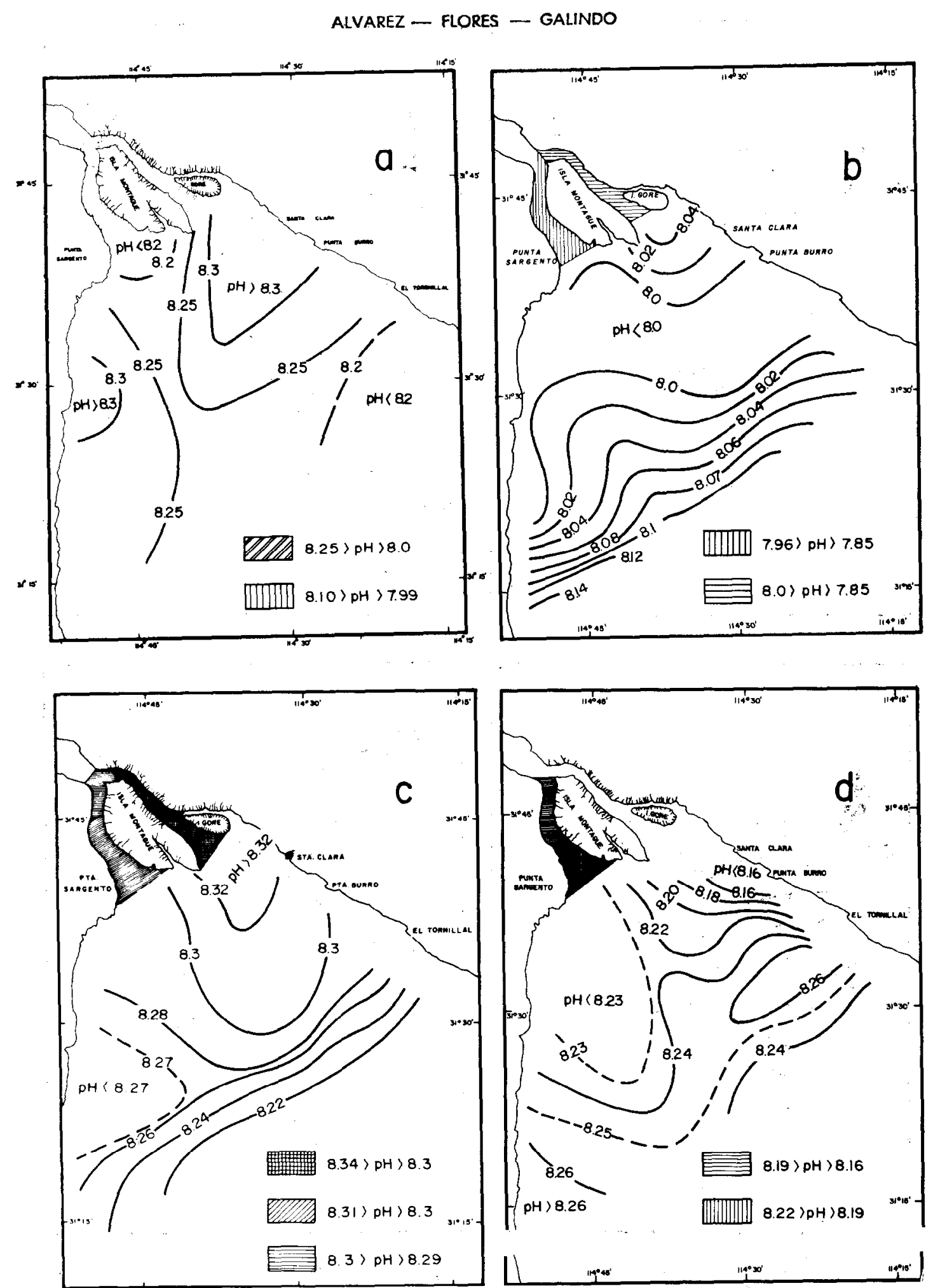

Fir. 8. Distribuciones Superficiales de pH pora: a. Febrero, b. Mayo, c. Junio, d. Julio. 
de diversas especies, algunas de las cuales deben encontrarse ya en situación ecológica desfavorable por la salinidad y temperatura extremadas. Sin embargo, es muy poco lo que se conoce sobre la ecología integral de la zona, desde el punto de vista biológico.

En la zana adyacente a Santa Clara, Son., las temperaturas y salinidades más elevadas fueron alrededor de $32^{\circ} \mathrm{C}$ y $36.50 \%$, respectivamente. Pero, si existe una corriente neta en el sentido contrario a las manecillas del reloj (Alvarez Borrego y Galindo Bect, 1974) en esta zona, el afluente de desecho que produciría la planta nuclear, se dirigiría hacia lo que fue la boca del Río Colorado.

En los días que precedieron al crucero de Marzo y durante el mismo, se registró cierta precipitación pluvial en nuestra área de interés. En la estación meteorológica de la Secretaría de Recursos Hidráulicos en Riitos, Son:, al norte del Golfo de Santa Clara, se registraron $3.9 \mathrm{~mm}$. y $3.4 \mathrm{~mm}$. de precipitación pluvial el 9 y 13 de Marzo respectivamente. En San Luis Río Colorado, Son., se registraron $7.1 \mathrm{~mm}, 11.7 \mathrm{~mm}$ y $5.0 \mathrm{~mm}$, el 9, 11 y 13 de Marzo respectivamente. En la estación de Presa Morelos, B. C., se registraron $14.0 \mathrm{~mm}$ el 9 de Marzo y en El Mayor, B. C., se registraron $4.0 \mathrm{~mm}$ el 9 de Marzo y lluvias inapreciables el 12 y 13 (Rep. de datos, Dirección de Hidr., Div. Ensenada, S.R.H.). Mal tiempo, con fuertes vientos que provocaban oleaje de hasta más de $1 \frac{1}{2} \mathrm{~m}$ de altura, pudo ser la causa de procesos intensos de mezcla responsables de la distribución un tanto irregular de la temperatura y salinidad en este crucero (Figs. $2 c$ y $4 c$ ). La precipitación pluvial registrada en el periodo de este crucero no fue suficiente para invertir el gradiente de salinidad, registrándose altas salinidades en la zona de isla Montague.

La distribución superficial de oxígeno depende de la temperatura, que regula el intercambio gaseoso con la atmósfera, y de los procesos de respiración y fotosíntesis; depende además, de procesos de mezcla y advección de masas de agua. La solubilidad del oxígeno en el agua de mar disminuye al aumentar la temperatura y también, en menor grado, al aumentar la salinidad. En los cruceros de
Enero y Febrero, se nota una alta correlación, con coeficiente negativo, entre la temperatura y el oxígeno fFigs. $2 a$ y b; $6 a$ y b); con oxígeno elevado donde la temperatura es baja, y viceversa. Esto indica que durante este período del año, la temperatura es el factor que controla la distribución superficial de oxígeno. En Mayo también se apreció una gran dependencia del oxígeno con la temperatura (Figs. $2 d$ y $6 d$ ). Al realizarse la reversión del gradiente de temperatura también se efectuó una reversión en el de oxígeno. Pero en Marzo y los meses de verano, la distribución de oxígeno fue afectada más fuertemente por otros factores. En Marzo (Fig 6c) posiblemente el factor más importante haya sido el oleaje provocado por los vientos registrados, mismo que causó una gran aereación. En este crucero se trabajó bajo condiciones meteorológicas muy adversas; el último día se estimó el oleaje en $1.7 \mathrm{~m}$, con gran turbulencia en la superficie. En los meses de verano y principios de ọtoño (Figs. 7a, b, c y d), la baja correlación de la distribución de oxígeno con la de temperatura posiblemente se deba a los efectos de fotosíntesis y respiración. Para corroborar ésto se necesitan datos sabre la productividad orgánica de la zona; sin embargo, los datos de pH apoyan el argumento de que son principalmente los factores biológicos los que están regulando la distribución de oxígeno en este período. La relación entre el oxígeno $y$ cl $\mathrm{pH}$ se establece a través de la fotosíntesis y la respiración que afectan el sistema del bióxido de carbono. Los datos de Junio (Figs. $3 a, 7 a$ y $8 c$ ) son los que muestran más claramente que la fotosíntesis y no la temperatura superficial, era la que estaba gobernando la distribución superficial de oxígeno en verano. Los valores más altos de oxígeno se registraron en la parte noreste, adyacente a la costa de Sonora (Fig. 7a), donde los valores de temperatura eran relativamente elevados, con respecto a los registrados en la parte sureste (Fig. 3a). Los valores de $\mathrm{pH}$ en la parte noreste fueron también los más elevados (Fig. 8c). La fotosíntesis debió haber sido más intensa en la parte noreste que en el resto de la zona de estudio, en Junio. Al realizarse la fotosíntesis se produce oxí- 
geno y se consume bióxido de carbono, aumentando el $\mathrm{pH}$.

Alvarez Borrego y Galindo Bect (1974) mencionaron que al estudiar la distribución supeficial de los parámetros hidrológicos se debe tomar en cuenta que el muestreo no se lleva a cabo simultáneamente en todas las estaciones hidrográficas, y por lo tanto las gráficas que se construyen con los datos son sólo una primera aproximación a la realidad. Cualquier interpretación de las mismas debe tomar en consideración los rangos de variación en funcion del tiempo, en el período de muestreo. Por esta razón se realizaron los estudios de variación diurna de Marzo y Mayo. Analizando las variaciones de cada una de las diferentes propiedades hidrológicas (Fig. $10 a$ y bl, en ciertas horas del día y comparándolas con variaciones de las mismas propiedades ocurridas a esas horas durante el descrrollo del crucero en el mismo mes, podemos saber si las distribuciones superficiales obtenidas son reales, o bien producto de la variación del tiempo durante el muestreo, en el traslado de una estación a otra. Variaciones de Marzo de $0.01 \%$ de salinidad, y $0.03 \mathrm{ml} / \mathrm{l}$ de oxígeno de las 11:00 a las 13:00 Hrs. (Fig. 10a), comparadas con variaciones horizontales de $0.36 \%$ de salinidad y $0.40 \mathrm{ml} / \mathrm{l}$ de oxígeno en el mismo período, de la estación 16 a la 12 tFigs. $1 a, 4 c$ y bcl; así como también variaciones de $0.02^{\circ} \mathrm{C}$ y $0.11 \%$ de las 13:00 a las 14:00 Hrs. (Fig. 10a), comparadas con las variaciones horizontales de $0.60^{\circ} \mathrm{C}$ y $0.50 \%$ en el mismo periodo de la estación 7 a la 13 /Figs. $1 a, 2 c$ y $4 c)$; indican que la variación encontrada horizontalmente a través del crucero de Marzo es significativamente real para el momento del muestreo; es decir, no es producto de los cambios ocurridos simplemente por la diferencia de hora en el muestreo. Sin embargo, una variación de $0.10 \mathrm{ml} / \mathrm{l}$ de oxígeno de las $13: 00$ a las 14:00 Hrs. (Fig 10a), y de $0.13 \mathrm{~m} ! / 1$ en el mismo tiempo, de la astación 7 a la 13 en la distribucion horizontal (Figs. la y $6 \mathrm{c}$ ), nos indica que la mayor parte de este cambio puede ser causado por la diferencia de hora en el muestreo. En el crucero de Mayo se encontró algo semejante al de Marzo, con variaciones de $0.26^{\circ} \mathrm{C}, 0.02 \% 00 y$ sin variación en el oxígeno, de las 12:50 a las 14:10 Hrs., comparadas con variaciones horizontales de $1.15^{\circ} \mathrm{C}, 0.29 \%$ y $0.21 \mathrm{ml} / \mathrm{l}$, en el mismo período de la estación 10 a la 9 (Figs. 1a, 2d, 4d y $6 d l$. Esto indica que las variaciones encontradas en el sentido horizontal, en Mayo, fueron tambien significativamente una función de la posición geográfica. Para obtener una mejor aproximación a la realidad, mediante un acercamiento a la simultaneidad de la toma de datos, sería necesario el contar con equipos más sofisticados. En el caso de la temperatura se podría utilizar equipo de rayos infrarrojos desde un avión que volara sobre la zona en unos pocos minutos. Para los demás parametros, la solución sería el colocar una serie de boyas con equipo automatizado, en las diferentes estaciones hidrográficas, trabajando al mismo tiempo, lo cual ha estado fuera de nuestro alcance económico.

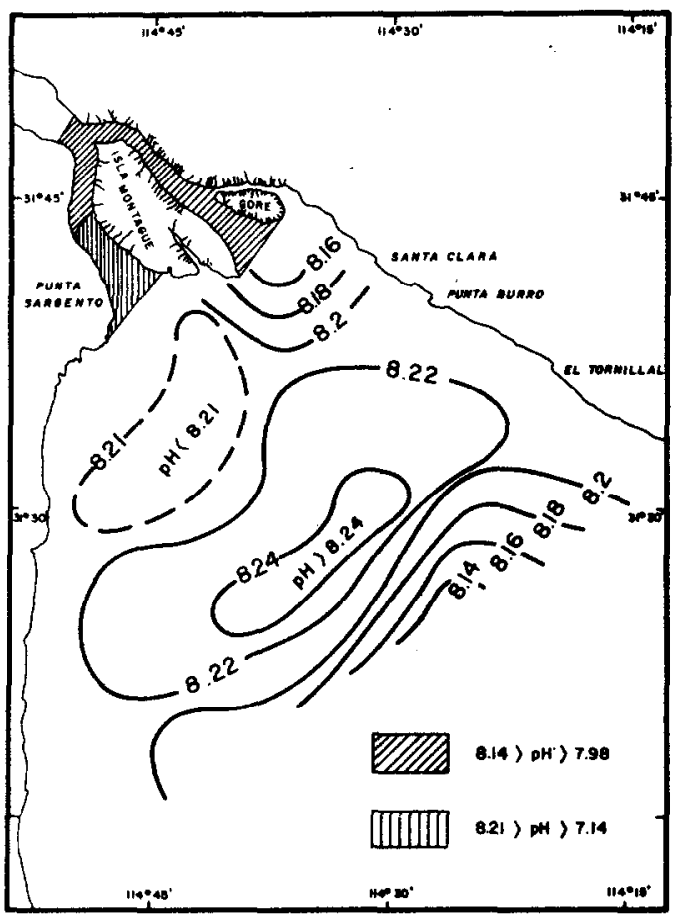

Fig. 9. Distribución superficial de $\mathrm{pH}$ para: Septiembre-Oclubre. 

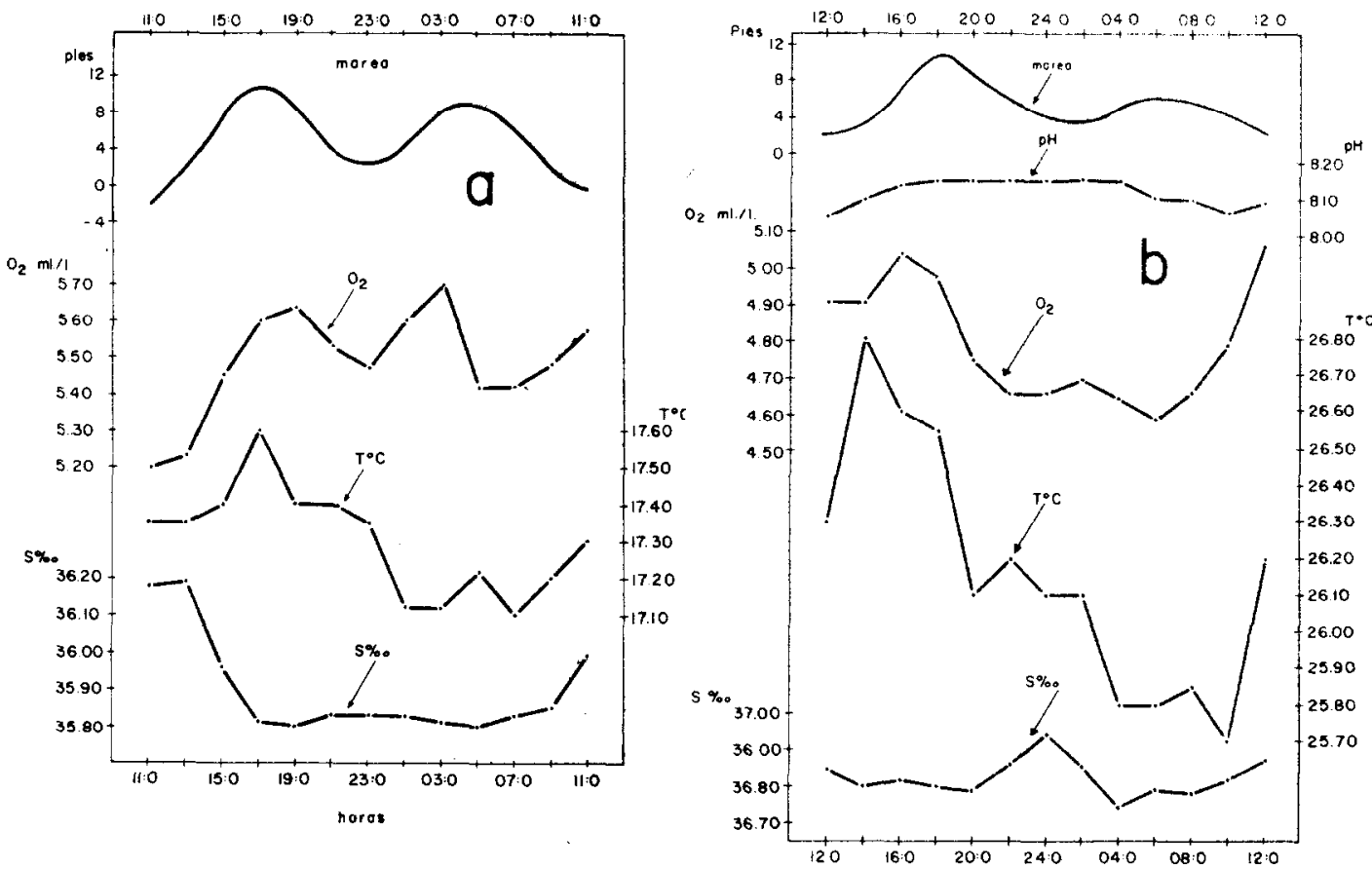

Fig. 10. Variación de Temperatura, Salinidod, Cancentración de oxigeno disuelto y pH durante un ciclo diurno. a. En Marzo (de las 11:00 del 9 de Marzo a las 11:00 del 10 de Marzol. b. En Mayo lde las 12:00 del 23 de Mavo a las 12:00 del 24 de Mayol.

En general se aprecia una más alta correlación de la temperatura con la variación diurna de la irradiacion solar (hora del día) que con el ciclo de marea (Fig. 10a y b). La salinidad muestra el efecto combinado de ambas, la marea y la irradiación solar, causante de la evaporacion, con una mayor correlación con la hora del día en Marzo (Fig. 10a), y una mayor correlación can la marea en Mayo (Fig. 10b). Esto se debe principalmente a que en Marzo el lugar escogido para el estudio de variación diurna (Estación A6, Fig. 1a) se encuentra bajo condicicnes mas oceánicas; $y$ en Mayo el lugar escogido se encuentra en aguas someras, frente a Santa Clara, Son., donde el efecto del flujo y reflujo de la marea es mayor. El máximo de salinidad de Mayo coincide con la marea baja a las 24:00 Hrs. (Fig. 10b), detectando en ese momento aguas provenientes de la zona circundante a isla Montague. La variación de oxígeno muestra también el efecto combinado de la marea y la irradiación solar, pero contrario a la sali- nidad muestra una mayor correlación con la marea en Marzo (Fig. 10a), y una mayor correlación con la hora del día en Mayo (Fig. 10b), con la concentración de oxígeno disminuyendo durante la nothe debido a la respiración.

\section{AGRADECIMIENTOS}

El presente trabajo constituye una parte de la tesis conjunta de Bernardo P. Flores Báez y Luis A. Galindo Bect, presentada a la Escuela Superior de Ciencias Marinas de la Universidad Autónoma de Baja California para obtener el título de Oceanólogo. Una versión donde se discuten los resultados para todo el año fue presentada ante el $V$ Congreso Nacional de Oceanografía en Guaymas, 1974. Se llevó a cabo como parte de un trabajo bajo contrato para la Dirección de Acuacultura de la Secretaría de Recursos Hidráulicos. Los autores hacen patente su agradecimiento al Ing. Daniel Muñoz Ferreira, Director de Acuacultura, por su disposición favorable para que sea 
publicado. Este trabajo fue además subvencionado parcialmente por la Institución Scripps de Oceanografía de la Universidad de California, debido a la colaboración desinteresada de Richard Schwartzlose a quien también le patentizamos nuestro agradecimiento. El Dr. John Hendrickson de la Universidad de Arizona y su grupo de trabajo, tuvieron una interacción continua con nosotros, la cual fue fructífera para ambos; permitiéndonos utilizar la embarcación "Adventyr" en la mayoría de los cruceros. Las cooperativas pesqueras de Puerto Peñasco, Son.: "Bahía Adair", "Pescadores Mexicanos", "Independientes", "Adolfo Ruiz Cortines" y "Punta Peñasco", colaboraron mediante el préstamo de dos barcos camaroneros para realizar los cruceros de verano y subvencionándolos parcialmente. La Universidad de Sonora colaboró permitiéndonos utilizar su embarcación para el crucero de octubre de 1973. Finalmente queremos agradecer la colaboración en los cruceros, de los compañeros Oceanólogos: Jorge Alberto Rivera. Manuel de Jesús Acosta Ruiz, Orlando Núñez Esquer, Jorge Ballesferos Grijalva, Marcos Miranda Aguilar, Manuel S. Galindo Bect, Federico Zambrano Martínez, Antonio Badán Dangon, José Luis Ortega Piñuelas, Horacio Haro Benútez, Luis García Pámanes, Antonio Diaz de León, al Geol. Michael Larroque, y al patrón del "Adventyr" David Cabrera Gonzólez.

\section{BIBLIOGRAFIA}

Alvarez Borrego, S., y L.A. Galindo Bect. 1974. Hidrología del Alto Golfo de Californio - 1. Condiciones durante Otoño. Ciencias Marinas, Vol. I, Núm. 1, Pag. 46-64.

División Ensenada. Dirección de Hidrología. Secretaría de Recursos Hidráulicos. Reporte de datos meteorolbgicos (no publicados).

Green, C. R. 1969. Meteorological conditions. En: Environmental impat of Brine Effluente on Gulf of California, U.S. Dep. Int., Res. and Dev. Prog. Rep. No. 387.

International Atomic Energy Agency study team United States-Mexico. 1968. Nuclear Power and Water Desalting Plants for Southwest United States and Northwest Mexico. NBS, U. S. Dept. Comm. 18 Pags.

Matthews, J. B. 1969. Tides in the Gulf of California. En: Environmental Impact of Brine Effluents on Gulf of California, U.S. Dept. Int. Res. and Dev. Prog. Rep. No. 387.

Matthews, J. B., D. A. Thornson y S. R. Browning. 1967. Tide Calendar for Puerto Peñasco, México, Tucson, Ariz. Univ. of Arizona, 14 Págs.

Meigs, P. 1953. World distribution of arid and semiarid homoclimates. En: Review of research on arid zone hydrology. (Maps dated 1952) UNESCO, Paris, Arid zone Programme, 1: 203-210.

Roden, G. I. 1964. Oceanographic aspects of Gulf of California. En: T. H. Van Andel y G. G. Shore, Jr., Eds., Marine Geology of the Gulf of California. Amer. Assoc. Petroleum Geologists, Memoir 3, Pag. 30-58.

Schreiber, Jr., J. F. 1969. Geographical and Geological features. En: Environmental Impact of Brine Effluents on Gulf of California, U.S. Dept. Int., Res. and Dev. Prog. Rep. No. 387.

Thompson, R. W. 1969. Tidal currents and general circulation. En: Environmental Impact of Brine Effluents on Gulf of California, U.S. Dept. Int., Res. and Dev. Prog. Rep. No. 387. 